\title{
MICROBIOLOGICAL PROFILE OF VAGINAL SWABS.
}

Sevitha Bhat, Nilica Devi, Shalini Shenoy

1. Assistant Professor, Department of Microbiology, Kasturba Medical College, Mangalore.

2. MSc Student, Department of Microbiology, Kasturba Medical College, Mangalore.

3. Professor, Department of Microbiology, Kasturba Medical College, Mangalore.

\section{CORRESPONDING AUTHOR}

Dr Sevitha Bhat,

Flat 102, Meadows,

Mannagudda Road, Ballalbagh,

Mangalore, Karnataka.

E-mail: sevitha@rediffmail.com,

Ph: 00919449831631.

ABSTRACT: BACKGROUND/OBJECTIVES: Bacterial vaginosis (BV), an alteration of vaginal flora involving a decrease in Lactobacilli and predominance of anaerobic bacteria, is the most common cause of vaginal complaints for women of childbearing age .Causative agents include Gardnerella vaginalis, facultative lactobacilli, Mycoplasma hominis, Viridans streptococci \& anaerobic bacteria. The most frequent manifestations of genitourinary candidiasis is vulvovaginal candidiasis \& is diagnosed in $40 \%$ of women with vaginal complaints. The present study was undertaken to study the occurrence of Bacterial vaginosis \& Vulvovaginal candidiasis in women with vaginal complaints. MATERIALS AND METHODS: The study included 100 High vaginal swabs with $\mathrm{H} / \mathrm{O}$ white discharge per vaginum for a period of 6 months from June 2011.Swabs were stained by Grams method \& were cultured. RESULTS: Out of 100 samples, in 35(35\%)Gram smear findings were normal \& no growth in culture. 26(26\%)samples had polymorphs with Gardnerella morphotype, gram negative curved rods, fusiforms \& Lactobacilli were absent/present in low numbers(1 to 2+), the smear was interpreted as consistent with BV. 15 samples (15\%) had the growth of Candida spp.

In the remaining 24 samples, the Gram stain findings had only vaginal epithelial cells with Lactobacilli, but the culture had the growth of Group B Streptococcus 10(10\%), E. coli \& coliforms 10(10\%),Enterococcus spp 14(14\%)which could just be colonisers.

CONCLUSION: Bacterial vaginosis is the predominant cause of leucorrhoea (26\%) followed by vulvovaginal candidiasis accounting for $15 \%$ of the cases. Detection of intrapartum vaginal colonisation of Group B Streptococci, E. coli \& coliforms \& Enterococcus spp. in pregnant women is of significance as it can get transmitted to the neonate $\&$ cause sepsis.

KEY WORDS : Bacterial vaginosis, Vaginal swabs, G. vaginalis

INTRODUCTION: Bacterial vaginosis (BV), an alteration of vaginal flora involving a decrease in Lactobacilli and predominance of anaerobic bacteria, is among the most common cause of vaginal complaints for women of childbearing age. It is well known that BV has an influence in acquisition of certain genital infections. This infection is characterized by a loss of indigenous (hydrogen peroxide-producing) Lactobacillus-predominant vaginal microflora, and a concurrent massive overgrowth of anaerobic bacteria. The most common include Gardnerella vaginalis, Mobiluncus species, Prevotella species (anaerobic bacteria), Mycoplasma hominis, and Atopobium vaginae ${ }^{1}$. At least $50 \%$ of patients have no symptoms. In the other half, it most often manifests clinically as a thin homogenous vaginal discharge, a vaginal $\mathrm{pH}>4.5$, presence 
of 'clue cells', and an amine odour after addition of $10 \%$ of potassium hydroxide. BV has been shown to increase the risk of obstetric and gynaecologic complications such as preterm labour and delivery, chorioamnionitis, post-caesarean endometritis, post-abortion pelvic inflammatory disease, and cervicitis ${ }^{1,3}$. Moreover, BV has been associated with many sexually transmitted infections (STIs), including infection with Chlamydia trachomatis, Neisseria gonorrhoeae, HSV-1 and 2, and an increased risk of HIV acquisition . Diagnostic criteria for BV included Nugent's method and culture.

The most frequent manifestations of genitourinary candidiasis include vulvovaginal candidiasis (VVC) in women, balanitis and balanoposthitis in men, and candiduria in both sexes. These diseases are remarkably common but occur in different populations, immunocompetent as well as immunocompromised. While VVC affects mostly healthy women, candiduria is commonly diagnosed in immunocompromised patients or neonates. In the majority of women, a diagnosis of VVC is made at least once during their childbearing years. Among the many causes of vaginitis, VVC is the second most common after bacterial vaginosis and is diagnosed in up to $40 \%$ of women with vaginal complaints in the primary care setting. A $10 \%$ potassium hydroxide $(\mathrm{KOH})$ preparation is more sensitive than a saline preparation in identifying yeast cells or hyphae ${ }^{2}$. Vaginal culture is the most accurate method for the diagnosis of VVC and is indicated if microscopy is negative 2 .

In pregnant women, Group B Streptococci (GBS) is a cause of cystitis, amnionitis, endometritis, and stillbirth. Occasionally, GBS has been a cause of endocarditis and meningitis, while in postpartum women; it has been identified as a cause of urinary tract infections (UTIs) and pelvic abscesses4. Prenatal screening for this condition is now established. Intrapartum colonisation of the mother's vagina with coliforms, enterococci is also associated with neonatal sepsis and meningitis in the newborn.

The present study was undertaken to study the occurrence of BV \& Vulvovaginal candidiasis in women with vaginal complaints.

MATERIALS AND METHODS: A retrospective study was undertaken . The study included 100 High vaginal swabs received in the laboratory from women in the age group 21-35 years who attended the hospital with $\mathrm{H} / \mathrm{O}$ white discharge per vaginum, abdominal pain and any other complaints formed the study population .The study was carried out in the Department of Microbiology, Kasturba Medical College, Mangalore for a period of 6 months from June 2011.

The swabs were stained by Grams method \& were cultured onto Thioglycollate broth, Mac Conkey Agar, Chocolate agar, Blood agar. The culture was incubated aerobically \& was examined after 18 to 24 hours $^{5}$.

Gram smear examination: Gram smear was evaluated for the presence of pus cells, vaginal epithelial cells, various bacterial morphotypes, clue cells \& yeasts .

Each microbial morphotype was quantitated under an oil immersion objective (1000 x) by using the following scheme: $1+(<1$ per field); $2+(1$ to 5 per field); $3+(6$ to 30 per field $) ; 4+($ $>30$ per field). Large gram-positive rods were taken as lactobacillus morphotypes; smaller gram-negative to gram-variable rods were considered as G. vaginalis and Bacteroidesspp 5 . As per table 1.

When the Lactobacillus morphotype was present alone or in combination only with the Gardnerella morphotype, the smear was interpreted as normal. When mixed flora, including not only the Gardnerella morphotype but also other gram-negative and gram-positive bacteria such as curved rods, gram-negative rods, fusiforms, and gram-positive cocci was present and when 
the Lactobacillus morphotype was absent or present only in low numbers (1 to 2+), the smear was interpreted as consistent with BV. The presence of morphotypes other than the Lactobacillus or Gardnerella morphotype was considered abnormal, regardless of quantity 5 .

The growth obtained on the culture plates was identified using conventional biochemical tests ${ }^{6}$. Group B Streptococcus was identified by Strep Pro grouping kit. \&Candida spp. were further identified by Germ tube test and CHROM agar.

RESULTS: Out of 100 samples, in 35(35\%) Gram smear findings were normal \& no growth in culture. $26(26 \%)$ samples had polymorphs with Gardnerella morphotype , gram negative curved rods, fusiforms\& Lactobacilli were absent/present in low numbers(1 to $2+$ ), the smear was interpreted as consistent with BV.

15 samples (15\%) had the growth of Candida spp.

In the remaining 24 samples, the Gram stain findings had only vaginal epithelial cells with Lactobacilli, but the culture had the growth of Group B Streptococcus 10(10\%), E.coli \& coliforms 10(10\%),Enterococcus spp 4(4\%) which could just be colonisers.

DISCUSSION: Bacterial vaginosis is characterized by a reduction in the prevalence and the numbers of hydrogen peroxide-producing lactobacilli and an increase in the concentration of Gardnerella vaginalis and resident anaerobic bacteria. Causative agents of BV thus include Gardnerella vaginalis, facultative lactobacilli, Mycoplasma hominis, Viridans streptococci \& anaerobic bacteria ${ }^{7}$.

It is associated with adverse outcomes related to upper genital tract and increased risk of HIV acquisition. An interesting observation is that new genital HPV infection in young women is associated with increased subsequent risk of developing BV. Other risk factors include multiple sexual partners and recent intercourse with a new partner, but metronidazole treatment of male partners has not reduced the rate of recurrence among affected women ${ }^{8}$.

The delicate balance of the vaginal ecosystem is challenged constantly by several factors such as hormonal changes, medications, intercourse, stress, infection, douching, and hygiene.

The unhealthy vaginal environment can be described as an imbalance in the vaginal bacterial ecosystem ${ }^{8}$.

At least $50 \%$ of patients were asymptomatic. In the other half, it most often manifests clinically as a thin homogenous vaginal discharge and presence of 'clue cells' 1,2 .

Although there are no universal standards, as a point of reference, the PHLS SOP recommends an High vaginal swabs from patients be Gram stained for BV and cultured for Trichomonas and yeasts.

We conducted a study on 100 cases of BV which were diagnosed by Gram stain and culture. It classifies gram stained vaginal smears into normal, intermediate and bacterial vaginosis based on the gram stain scoring system.

In our study, out of the 100 smears examined , 26 smears(26\%) were interpreted as consistent with BV. 15 samples (15\%) had the growth of Candida spp and is comparable to previous studies where bacterial vaginosis is common compared to VVC in women with vaginal complaints in the primary care setting 2 .

Culture is not reliable for diagnosis of most of the bacterial diseases; as the organisms which are involved in BV cannot be isolated in the laboratory easily and as normal women also have this flora in their vagina in small numbers . 
Culture was used to detect the vaginal colonisation with coliforms, Group B Streptococcus, Enterococcus species which is of importance in pregnant women .

CONCLUSION: BV is the predominant cause of leucorrhoea (26\%) followed by vulvovaginal candidiasis accounting for $15 \%$ of the cases. Detection of intrapartum vaginal colonisation of Group B Streptococci, E. coli \& coliforms \& Enterococcus spp. in pregnant women is of significance as it can get transmitted to the neonate \& cause sepsis.

ACKNOWLEDGEMENT: The authors are grateful to Manipal University for providing the facilities to perform the study.

Table 1: Nugent's method of diagnosis of bacterial vaginosis

\begin{tabular}{|c|c|c|c|}
\hline$*$ Score & $\begin{array}{c}\text { Lactobacillus } \\
\text { Morphotypes }\end{array}$ & $\begin{array}{l}\text { G. vaginalis and } \\
\text { Bacteroides spp. } \\
\text { Morphotypes }\end{array}$ & $\begin{array}{c}\text { Curved gram } \\
\text { variable rods }\end{array}$ \\
\hline 0 & $4+$ & 0 & 0 \\
\hline 1 & $3+$ & $1+$ & $1+$ to $2+$ \\
\hline 2 & $2+$ & $2+$ & $3+$ to $4+$ \\
\hline 3 & $1+$ & $3+$ & \\
\hline 4 & 0 & $4+$ & \\
\hline
\end{tabular}

Table 2: Gram smear and culture findings of $100 \mathrm{High}$ vaginal swabs

\begin{tabular}{|l|c|}
\hline & Number and percentage \\
\hline $\begin{array}{l}\text { Normal( Gram smear findings were normal \& } \\
\text { no growth in culture) }\end{array}$ & $35(35 \%)$ \\
\hline $\begin{array}{l}\text { BV\{ Gardnerella morphotype, gram negative } \\
\text { curved rods, fusiforms\& Lactobacilli were } \\
\text { absent/present in low numbers(1 to 2+)\} }\end{array}$ & $26(26 \%)$ \\
\hline Candida & $15(15 \%)$ \\
\hline $\begin{array}{l}\text { Colonisers (Gram stain findings had only } \\
\text { vaginal epithelial cells with Lactobacilli, but } \\
\text { the culture had the growth of Group B } \\
\begin{array}{l}\text { Streptococcus, E. coli \& coliforms, } \\
\text { Enterococcus spp. ) }\end{array}\end{array}$ & $\begin{array}{l}\text { Group B Streptococcus 10(10\%) } \\
\text { E. coli\& coliforms 10(10\%) } \\
\text { Enterococcus spp 14(14\%) }\end{array}$ \\
\hline
\end{tabular}




\section{REFERENCES:}

1. Morris M, Nicoll A, Simms I, et al.: Bacterial vaginosis: a public health review. BJOG 2001, 108:439-450.

2. Jacqueline M. Achkar and Bettina C. Fries .Candida Infections of the Genitourinary Tract. Clin. Microbiol. Rev 2010; 23 (2): 253-273.

3. Rao PS, Devi S, Shriyan A, Rajaram M, Jagdishchandra K. Diagnosis of bacterial vaginosis in a rural set up: comparison of clinical algorithm, smear scoring and culture by semiquantitative technique. Indian J Med Microbiol2004; 22(1):47-50.

4. Ugwumadu AH: Bacterial vaginosis in pregnancy. CurrOpinObstetGynecol 2002, 14:115118

5. Udayalaxmi, GopalkrishnaBhat, SubbannayyaKotigadde, ShaliniShenoy Comparison of the Methods of Diagnosis of Bacterial Vaginosis . Journal of Clinical and Diagnostic Research. 2011 June, Vol-5(3): 498-501.

6. MacFaddin, J.F. Biochemical Tests for Identification of Medical Bacteria, 2nd ed., Baltimore: Williams and Wilkins, 1980.

7. Mohanty S, Sood S, Kapil A, Mittal S. Interobserver variation in the interpretation of Nugent scoring method for diagnosis of bacterial vaginosis. Indian J Med Res 2010;131:88-91.

8. Verstraelen $H$, Verhelst $R$ : Bacterial vaginosis: an update on diagnosis and treatment.Expert Rev Anti Infect Ther 2009, 7:1109- 1124. 